American Journal of Animal and Veterinary Sciences 7 (1): 28-35, 2012

ISSN 1557-4555

(C) 2012 Science Publications

\title{
An Induced Hematoma in Caudoputamen Nuclei in Rats Causes Central Pain when the Thalamus is also Implicated and the Central Sensitization is Reversed with Gabapentin
}

\author{
Lema, P.P. and P. Vachon \\ Department of Veterinary Biomedicine, \\ Faculty of Veterinary Medicine, University of Montreal, \\ St-Hyacinthe, Quebec, Canada
}

\begin{abstract}
Problem statement: The objective of this study was to evaluate pain sensitisation in rats following the induction of an intracerebral hemorrhage by injecting a collagenase solution in the caudoputamen nucleus of the right basal ganglia and to evaluate gabapentin as an analgesic for central pain. Approach: Thirty male Sprague-Dawley rats weighing between 175-300 g were used. In a first experiment, 3 groups of 6 animals were used to evaluate pain threshold using the Hargreaves test (thermal sensitivity) only. Following 3 days of behavioral testing (baseline values), animals in each group were injected intracerebrally either with $0.5,1$ or $2 \mu \mathrm{L}$ of a collagenase solution $\left(0.5 \mathrm{U}_{2} \mu \mathrm{L}^{-1}\right.$ Type VII collagenase) inducing a hematoma in the right caudoputamen nucleus and/or thalamus. They were then tested for the next 9 consecutive days. In a second experiment, gabapentin was evaluated for the reversal of thermal hyperalgesia and mechanical allodynia (using von Frey filaments) following the intracerebral injection of $3 \mu \mathrm{L}$ of the collagenase solution. Results: No pain-related behavioral changes were observed following injections with 0.5 and $1 \mu \mathrm{L}$ of the collagenase solution. However with $2 \mu \mathrm{L}$, reaction times were significantly faster on days 3-7 in the right and left hind paws compared to baseline values. The lesion was localized only in the caudoputamen nucleus for animals receiving 0.5 and $1 \mu \mathrm{L}$ of collagenase whereas lesions extended in the ipsilateral thalamic nuclei (lateral-dorsal and lateral-posterior nuclei) for animals receiving $2 \mu \mathrm{L}$ of collagenase. Gabapentin reversed mechanical allodynia and thermal hyperalgesia in animals with caudoputamen and thalamic lesions. Conclusion: These preliminary results suggest that central pain was induced in rats with a collagenase-induced intracerebral hemorrhage localized in the thalamus and that mechanical allodynia and thermal hyperalgesia were reduced with gabapentin treatment.
\end{abstract}

Keys words: Central pain, ganglia and thalamus, intracerebral hematoma, Sprague-Dawley rats, gabapentin treatment, intracerebral hemorrhage, collagenase solution, caudoputamen nucleus, thermal hyperalgesia, mechanical allodynia, thalamic lesions

\section{INTRODUCTION}

Intracerebral hemorrhage, mainly caused by trauma and stroke in humans, is one of the principal causes of incapacitation and death in the population of industrialized countries (Reilly and Bullock, 1997; Graham and Gennarelli, 1997). Cerebrovascular accidents often cause an intracerebral hemorrhage leading to severe and debilitating neurological symptoms and they are the 3rd cause of mortality in the United States, following cardiovascular disease and cancer (Reilly and Bullock, 1997). Central pain has been well documented in humans. Causes include stroke, multiple sclerosis, Parkinson's disease, spinal cord injury and brain trauma and the main syndrome has been identified as central post-stroke pain (Andersen et al., 1995).

Traumatic cerebral hemorrhages are also seen in veterinary medicine. Nuclear magnetic resonance has shown that dogs may suffer from cerebrovascular accidents leading to brain hemorrhage (Platt and Garosi, 2003). Primary and secondary hypertension in dogs predisposing to intracranial hemorrhage (Dukes, 1992) is known to occur with hypothyroidism and hyperlipoproteinemia (Thomas, 1996). Diseases such as sepsis, coagulopathy, neoplasia and heartworms are also associated with cerebral infarction in dogs (Platt and Garosi, 2003). Therefore, different pathologies in

Corresponding Author: P. Vachon, Department Veterinary Biomedicine, Faculty of Veterinary Medicine,

University of Montreal, St-Hyacinthe, Quebec, Canada Tel: (450) 773-8521 ext. 8962 Fax: (450) 778-8109 
veterinary medicine of companion animals cause intracerebral hemorrhaging; however, little is known about the development and perception of pain associated with these cerebral insults. Cats and monkeys have been used in research to understand the role of central nervous system structures in the production of central pain (Craig, 1998). A recent paper has shown that central pain occurs in rats following lesions in the thalamus (Wasserman and Koeberle, 2009). Most animal models of central pain had previously been based on spinal cord and brain stem injury (Wall and Melzack, 2001) obtained by weight drops (Anderson 1982; Greenberg et al., 1978), compression (Tarlov, 1972), crushing (Rivlin and Tator, 1978), photochemically induced injury (Hao et al., 1992; Watson et al., 1986), excitatory neurotoxin methods (Gorman et al., 2001; Wilcox, 1988) and spinal hemisection (Bennett et al., 2000; Christensen et al., 1996; 1997).

The objective of the present studies was to evaluate if central pain occurs following the induction of an intracerebral hemorrhage in rats using the Rosenberg model where the lesion in mainly in the caudoputamen nuclei since central post-stroke pain has been shown with lesions to the basal ganglia in humans (Kim, 2003). Following the induction of the intracerebral hemorrhage in rats, two behavioral tests were used to evaluate central pain, the Hargreaves test and von Frey filaments used to evaluate thermal hyperalgesia and mechanical allodynia respectively. To confirm that related changes that occurred with brain trauma were related to pain, gabapentin, a commonly used central analgesic for the treatment of neuropathic and central pain, was evaluated.

\section{MATERIALS AND METHODS}

Animals: Thirty male Sprague-Dawley rats (Crl:CD (SD) IGS BR stock; Charles River, St-Constant, Qc, Canada) weighing between 175-300 g were used in this study. Following arrival, they were housed in a standard environment (fresh filtered air: 15 changes $\mathrm{h}^{-1}$, temperature: $21 \pm 3^{\circ} \mathrm{C}$, humidity: $40-60 \%$ and light-dark cycle: $12 \mathrm{~h}: 12 \mathrm{~h})$. They were pair-housed in polycarbonate cages (Ancare, Bellmore, NY, USA) on hardwood bedding (Beta chip, Northeastern Products Co., Warrenburg, NY, USA) and acclimated to their environment for 7 days prior to the initiation of the study. Rats received tap water and rodent chow (Charles River Rodent Chow 5075, St-Constant, Qc, Canada) ad libitum. The University of Montreal's Faculty of Veterinary Medicine Institutional Animal Care and Use Committee approved the experimental protocol prior to animal use in accordance with the guidelines of the CCAC (1993).
Experimental design: For the first experiment, eighteen intact rats were trained for 5 days on the Hargreave's apparatus only. Behavioral testing was then performed for 3 days to obtain thermal sensitivity baseline values. Rats $(n=6 /$ collagenase dose) were then injected intracerebrally with $0,5,1$ or $2 \mu \mathrm{L}$ of a collagenase solution $\left(0.5 \mathrm{U} 2 \mu^{-1}\right)$ to produce a hematoma centered in the caudoputamen nuclei (basal ganglia) in the right cerebral hemisphere. Following the surgery, thermal sensitivity of both left and right hind paws was determined daily using the Hargreaves test for 9 consecutive days. Hargreaves test evaluates thermal sensitivity to a progressive intensity of heating light source (Plantar test Hargreaves method, IITC Life Sciences, Series 8, Model 390, Woodland Hills, CA). The beam of light was applied on the left and right hind paws and animals voluntarily retracted their hind paw from the light source upon reaching the threshold of intolerable pain sensation. The maximum duration over which the hind paw was exposed was set at $20 \mathrm{sec}$. Reaction times were taken on three occasions, with a 20 min delay between trials and the daily reported value for each animal was the mean of 3 trials for both the right and left hind paw. Rats were acclimated to the apparatus for $20 \mathrm{~min}$ prior to each daily testing.

In the second experiment, a group of animals $(n=$ 12) underwent training for 5 days on the Hargreaves test and with von Frey filaments. Following training, baseline values were recorded for 3 days. Hargreaves test was performed in a similar fashion to the first experiment of this study. Von Frey filaments were used to evaluate mechanical sensitivity according to a previously published method (Vachon et al., 2004). Briefly, paw withdrawal thresholds were evaluated on the plantar surface of the right and left hind paw using von Frey monofilaments (0.4-30 g; Stoelting, Wood Dale, IL, USA). Monofilaments were applied for $2 \mathrm{sec}$ in the central region of the plantar surface. The threshold was taken as the lowest force that caused at least 3 withdrawals out of 5 consecutive stimuli. Values reported are the threshold force in grams corresponding to the hind paw withdrawal. Mechanical sensitivity was evaluated first and then thermal hyperalgesia and the acclimation period to both apparatus was $20 \mathrm{~min}$ prior to testing. Following the collection of baseline data, rats were injected intracerebrally with $3 \mu \mathrm{L}$ of a collagenase solution $\left(0.5 \mathrm{U} 2 \mu \mathrm{L}^{-1}\right)$ to produce a hematoma centered in basal ganglia of the right cerebral hemisphere. Both tests were to be performed once on the 2nd day following surgery to establish that neuropathy was present and then twice daily (once without and once with treatment) on subsequent days for the treatment group $(n=6)$ and saline gavage control group $(n=6)$. 
Treated animals received $100 \mathrm{mg} \mathrm{kg}^{-1}$ gabapentin (Nakazato-Imasato et al., 2009) by gavage (100 mg $\mathrm{mL}^{-1} ; 1 \%$ methylcellulose solution) $30 \mathrm{~min}$ prior to behavioral testing. For both experiments, the person doing the evaluations was blinded.

Prior to and following the intracerebral collagenase injection, animals were evaluated daily, prior to manipulations, with selected motor behavioral tests that have previously been described (Lema et al., 2004; 2005). This exam consisted of general activity, evaluated as the immediate exploration of the immediate environment when the animal was placed on a novel hard surface and locomotion (linear locomotion or unilateral rotation), observed at the same time. Paresis was evaluated with visual positioning by holding the rat above a hard surface and lowering it toward a hard surface. A normal response consists in a full extension and reaching for the hard surface. Tail rigidity was evaluated by simply elevating it at midlength and observed for flexibility or rigidity.

Surgical methods: The surgical procedures have been previously described (Lema et al., 2004; 2005). Following the induction and maintenance of anesthesia with isoflurane (Aerrane, Baxter, Mississauga, ON, Canada), rats were placed in a stereotaxic instrument (David Kopf Instruments, Tujunga, CA, USA). A sagittal skin incision was made and a burr hole $(1.5 \mathrm{~mm}$ diameter) was drilled in the bone at the following stereotaxic coordinates: antero-posterior $0.0 \mathrm{~mm}$ and lateral $3.0 \mathrm{~mm}$, in reference to bregma, so that the injection site would be in the right caudoputamen nuclei (Paxinos and Watson, 1998). To induce the hematoma, a collagenase solution composed of $2.5 \mathrm{U}$ collagenase Type VII (Sigma-Aldrich, Oakville, ON, Canada) in 10 $\mu \mathrm{L}$ saline (Abbott Laboratories, St-Laurent, QC, Canada) was injected over a period of $10 \mathrm{~min}$ via the burr hole in the right caudoputamen nucleus, $6 \mathrm{~mm}$ below the dura mater with a $5 \mu \mathrm{L}$ Hamilton syringe. The needle was removed $5 \mathrm{~min}$ following the end of the injection and the skin was sutured. During the surgery body temperature was monitored with a rectal thermometer (Thermalert TH-8, Physitemp, Clifton, NJ, USA) and it was maintained within normal limits (35.5-37. $\left.5^{\circ} \mathrm{C}\right)$ by keeping animals on an electric heating pad. Following surgery, no analgesics were administered and animals were maintained under a heating lamp until recovery. Since most analgesics have anti-inflammatory properties (Ferland et al., 2007), no analgesics were administered postoperatively since this could change the outcome of the study. We also wanted to reproduce the Rosenberg model in which no analgesic is administered either pre- or post-operatively (Rosenberg et al., 1990).
Histological methods: Following the completion of the behavioral testing animals were anesthetized to a surgical plane using pentobarbital (100 mg kg-1, IP) (MTC Pharmaceuticals, Cambridge, Ontario, Canada). They were then perfused through the heart, first with a physiological dextrose-sucrose solution $\left(100 \mathrm{~mL} \mathrm{rat}^{-1}\right.$; solution composition (1L): $8 \mathrm{~g} \mathrm{NaCl}, 4 \mathrm{~g}$ dextrose, $8 \mathrm{~g}$ sucrose, $0.23 \mathrm{~g}$ calcium chloride) followed by a $10 \%$ buffered formalin solution $\left(100 \mathrm{~mL} \mathrm{Rat}^{-1}\right)$. All chemicals used for perfusion solutions were ordered from Sigma (Sigma Inc., St-Louis, MO, USA). For microscopic evaluation, brains were fixed in formalin for $48 \mathrm{~h}$ and embedded in paraffin. Five $\mu \mathrm{m}$ horizontal sections were taken and stained with a hematoxylin, eosin, phloxin and saffron stain. All lesions were confirmed by a certified veterinary pathologist (Pierre Helie DVM, DACVP, Department of Veterinary Pathology, University of Montreal).

Statistical analysis: An analysis of variance using a linear model with repeated measures and post hoc Tukey tests were used to assess whether the changes in the Hargreaves and von Frey tests were significantly different form pre-operative values. For the analysis of variance time as the intersubject factor and treatment as the intrasubject factor were used. Statistical analyses were carried out with SAS (Version 9.1, Cary, N.C.). The level of significance was set at 0.05 .

\section{RESULTS}

Daily motor behaviors observed following the induction of cerebral lesions were decreased exploration of the immediate environment, unilateral circling, hemiparesis of the right anterior right limb and tail rigidity which were more pronounced in animals that received 2 and $3 \mu \mathrm{L}$ of the collagenase solution (0.5 U $2 \mu \mathrm{L}^{-1}$ ). These are typical motor changes observed following the induction of a hematoma in the caudoputamen nucleus (Lema et al., 2004; 2005).

In the first experiment, animals that received 0,5 and $1 \mu \mathrm{L}$ of collagenase intracerebrally showed no change on the Hargreaves test when comparing pre and post surgery reaction times (results not shown). For rats receiving $2 \mu \mathrm{L}$ of collagenase, reaction times for both right and left hind paws were significantly decreased on days $3,4,5,6(\mathrm{p}<0.001)$ and $7(\mathrm{p}<0.01)$ compared to baseline values (Fig. 1). These rats returned to baseline reaction times on days 8 and 9 ( $\mathrm{p}=$ n.s.). Photomicrographs of histological sections taken at $48 \mathrm{~h}$ post collagenase-induce lesions are shown in Fig. 2. 


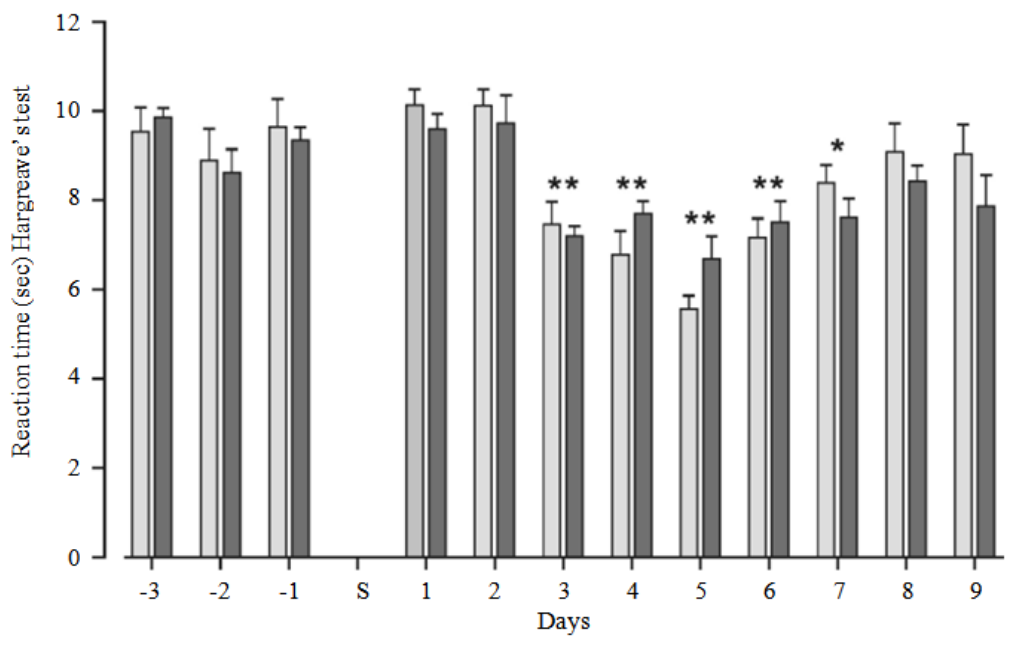

Fig. 1: Histogram representation of the mean $( \pm \mathrm{SE})$ thermal sensitivity (Hargreave's test) results in male SpragueDawley rats (right hind paw/light gray, left hind paw/dark) prior to (Days -3, -2, -1) and following (Days 1-9) the stereotaxic injection of $2 \mu \mathrm{L}$ of a collagenase solution $(0.5 \mathrm{U} / 2 \mu \mathrm{L})$ in the right caudoputamen nucleus (Surgery). $* * \mathrm{p}<0.001, * \mathrm{p}<0.01$

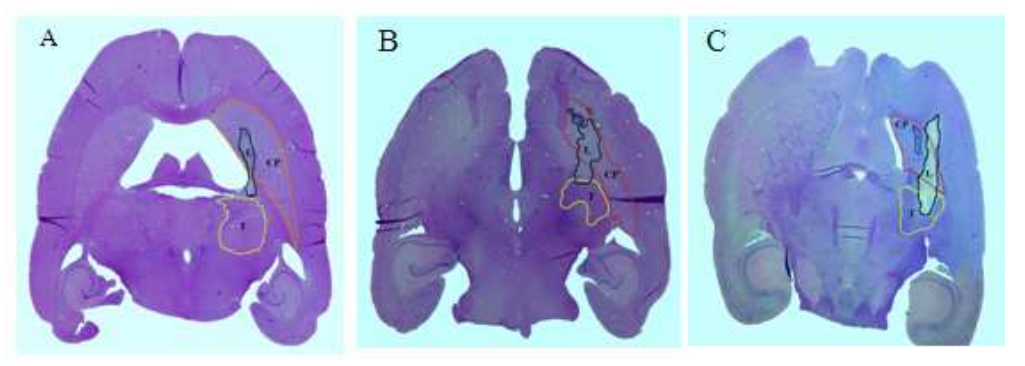

Fig. 2: Extent of the intracerebral hemorrhage in male Sprague-Dawley rats following stereotaxic injections of 0.5 $\mu \mathrm{L}$ (A; Group 1), $1 \mu \mathrm{L}$ (B; Group 2) and $2 \mu \mathrm{L}$ (C; Group 3) of a collagenase solution $(0.5 \mathrm{U} / 2 \mu \mathrm{L}$ ) in the caudoputamen nucleus. L lesioned area $\mathrm{CP}$ caudoputamen nucleus $\mathrm{T}$ thalamic nuclei

The lesion was mainly localized in the caudoputamen nucleus for animals receiving 0.5 and $1 \mu \mathrm{L}$ of collagenase. Lesions of the internal capsule were observed in all groups. Animals receiving $2 \mu \mathrm{L}$ of collagenase intracerebrally had lesions that extended to the ipsilateral thalamic nuclei mainly the lateral-dorsal and lateral-posterior nuclei.

The effect of gabapentin on allodynia (von Frey filaments) and thermal hyperalgesia (Hargreaves test) are presented in Fig. 3A and B respectively. On the second and third day following the surgery, pain thresholds were decreased on both von Frey and Hargreaves tests $(p<0.01)$. However following the administration of gabapentin $\left(100 \mathrm{mg} \mathrm{kg}^{-1} \mathrm{PO}\right)$ on the 3rd day, pain thresholds were not different from baseline values for both tests. On the fourth day, prior to gabapentin treatment, animals did not show allodynia or hyperalgesia which suggests a residual effect of treatment. Since no difference was noted on the 4th day, when compared to baseline values, animals were not treated with gabapentin on the 4th post surgical day. The lesion was more extensive in the caudoputamen nucleus and in the lateral-dorsal and lateral-posterior nuclei of the thalamus (lesion not shown). For the control group, mechanical allodynia and thermal hyperalgesia persisted on the 2nd, 3rd and 4th day following the collagenase injection.

As previously shown in other similar studies (Lema et al., 2004; 2005), clear spaces interpreted as edema, extended in the corpus callosum and into the contralateral basal ganglia and thalamus following the administration of 2 and $3 \mu \mathrm{L}$ of the collagenase solution. This may explain the bilateral effects on central pain induction. 
American J. Animal \& Vet. Sci., 7 (1): 28-35, 2012

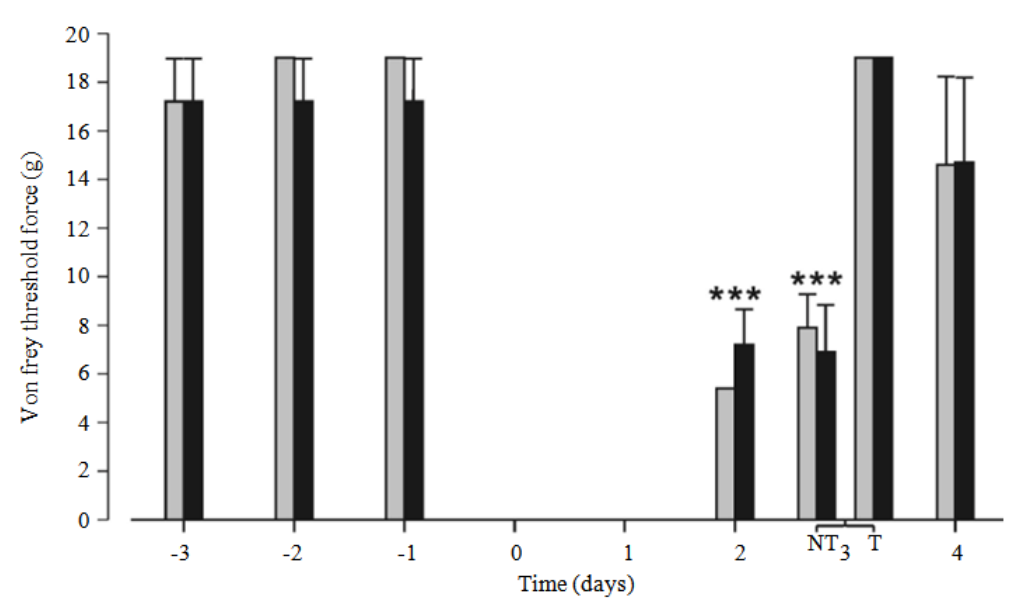

(a)

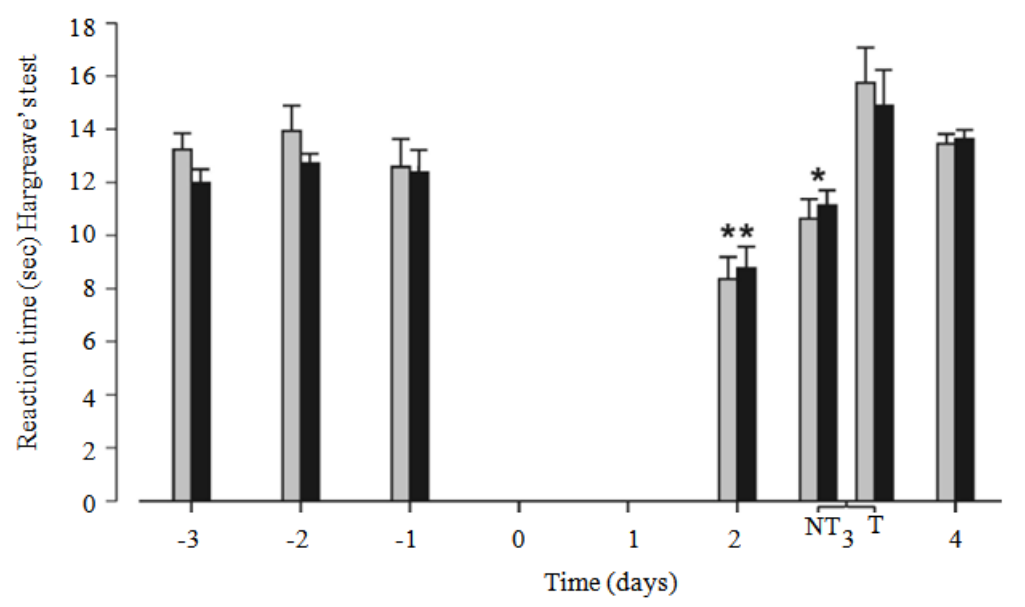

(b)

Fig. 3: Effects of gabapentin (100 mg kg $\left.\mathrm{m}^{-1}\right)$ on von Frey (A) and Hargreaves (B) tests in male Sprague-Dawley rats (right hind paw/light gray, left hind paw dark ${ }^{-1}$ ) following a stereotaxic injection of $3 \mu \mathrm{L}$ of a collagenase solution $(0.5 \mathrm{U} / 2 \mu \mathrm{L})$ in the caudoputamen nucleus. No significant changes from baseline occurred in control animals receiving saline by gavage. (Data not shown) $* * * \mathrm{p}<0.001, * * \mathrm{p}<0.01, * \mathrm{p}<0.05$

\section{DISCUSSION}

Animal models have played an important role in elucidating the cascade of cellular and biochemical events occurring after traumatic brain injury. Previous studies have reported traumatic brain injury with models such as injections of autologous blood, balloon distension, inertial acceleration, force impact and cold (Finnie and Blumberg, 1990). Rosenberg et al. (1990) have established the collagenase-induced intracerebral hematoma model, which is highly reproducible and shows many characteristics of the intracerebral hemorrhagic process in mammals. Only one recent paper has shown that collagenase-induced thalamic lesions can cause central pain in rats (Wasserman and
Koeberle, 2009). Since we had experience with the Rosenberg model and that they brain lesion caused by the collagenase injection in the basal ganglia may also affect the thalamus, we decided to evaluate pain perception in this model.

Results show that central pain, when evaluated with the Hargreaves and von Frey filaments tests, was observed following the production of an intracerebral hematoma when the collagenase injection is in the basal ganglia. However central pain sensitization was noted only in animals where the lesion extended in the posterior thalamus and not when lesions occurred in the caudoputamen nucleus only. When using this traumatic brain injury model, previous studies (Lema et al., 2004; 2005) have shown that animals had poor motor 
coordination and a deficit in motor initiation. Since the basal ganglia plays a role in the initiation of movement and postural control, animals would be expected to have increased response times when evaluated on reaction time tests. However, results show a decreased reaction time to a thermal painful stimulus suggesting the establishment of central pain. In Huntington's disease, where there is a neurodegenerative process of the basal ganglia, reflexes are usually decreased although muscle rigidity is present (Kandel et al., 1991). We therefore do not attribute the results to a change in reflex excitability at the level of the spinal cord. Also, in our experiment, normal responses were seen with von Frey and Hargreaves tests following gabapentin treatment in animals with the induced hematoma and these findings are highly suggestive of a central analgesic effect. We chose gabapentin as a central analgesic since it has been identified as part of the firstline medications for the treatment of neuropathic pain by the International Association for the Study of Pain (Dworkin et al., 2007). However in a recent review, gabapentin was suggested as a second-line treatment for central post-stroke pain in humans (Kumar et al., 2009).

Lesions in the basal ganglia or thalamus will cause motor and sensory problems in the contralateral side of the lesion. In our study, animals showed pain-related behaviors in both hinpaws. In the model established by Wasserman and Koeberle (2009) the total amount of collagenase injected was only $0.025 \mathrm{U}$ and pain sensation was altered only on the contralateral side. We injected $0.125,0.25$ and $0.5 \mathrm{U}$ in the first experiment and $0.75 \mathrm{U}$ in the second experiment. Lesions were therefore much more extensive than those seen in the experiments by Wasserman and Koeberle (2009). Animals that received 0.5 and $0.75 \mathrm{U}$ had lesions that were not only localized on the ipsilateral side but edema was observed all along the corpus callosum and in the contralateral basal ganglia and thalamus. This could very well explain why pain-related behaviors observed in both hindpaws and not only unilaterally demonstrated in the study by Wasseman and Koeberle (2009). On study has shown that unilatereal thalamic lesions can in some instances cause a bilateral pain syndrome in humans (Kim, 1998).

To better characterize this model, behavioral evaluations should be performed for a longer duration of time (weeks or months) and should evaluate different pain modalities other than mechanical allodynia with von Frey filaments and thermal hyperalgesia with the Hargreaves test. Compared to our previous results where the reaction time on the Hargreaves decreased to $5 \mathrm{sec}$ in neuropathic animal with the sciatic nerve ligation model, the animals with the caudoputamen- thalamus lesions had a longer reaction time (7-9 sec) following the $2 \mu \mathrm{L}$ collagenase solution injection which suggests that the pain is not as severe in these animals (Guenette et al., 2007; Beaudry et al., 2010). However with the $3 \mu \mathrm{L}$ collagenase injection, pain threshold were similar to the animals with sciatic nerve injury. Our results also show that animals appeared more affected on mechanical allodynia than thermal hyperalgesia. Interestingly it is also common in humans to have a very severe pain, mainly a mechanical allodynia, following central lesions such as a stroke (Kumar et al., 2009).

Lesions at any level of the neuraxis can cause central pain when pain pathways and associated structures are implicated. Lesions in the dorsal horn of the spinal cord, ascending spinothalamic pathways, brain stem, thalamus, subcortical white matter and cerebral cortex have all been reported to cause central pain (Bowsher, 1996; Bowsher et al., 1998; Cassinari and Pagni, 1969; Garcin, 1968; Leijon, 1989; Fishman et al., 2009). Lesions that produce central pain have best been studied in central post-stroke pain and it was found that $60 \%$ of lesions engage the thalamus (Bowsher, 1996). Hemorrhages in the basal ganglia produce sensory symptoms such as dysesthesia, mainly sensory deficits (Bowsher et al., 1998; Kim, 2003; Siddal et al., 2003). Pain associated with lesions of lenticulo-capsular nuclei is associated with lesions in the posterior limb of the internal capsule (Kim, 2003) and thalamus (Groothuis et al., 1977). Loss of lateral thalamic substrate results in the disinhibition or release of the medial thalamus, which is thought to be important for the emotional aspect of pain (Craig, 1998). In the present study, lesions in the posterior thalamus were also seen in the rats with highest volume of intracerebrally injected collagenase (2 and $3 \mu \mathrm{L})$. Interestingly, pain was noted only on the $3^{\text {rd }}$ day following the lesion following the collagenase injection. Post-stroke pain usually develops some time after the brain insult in 10-35\% of human patients and the time of onset to pain may be relatively rapid (within 1 month) (Andersen et al., 1995; Hansen, 2004; Widar et al., 2002). With spinal cord injuries onset time may be as long as 2 years (Siddal et al., 2003). In most cases central pain tends to persist and it is constantly present, with no pain-free intervals. However, in some cases, pain free intervals occur a few hours a day only.

\section{CONCLUSION}

A transient central pain was induced in rats with an intracerebral hemorrhage localized in the caudoputamen and thalamic nuclei following the stereotaxic injection of collagenase. The contralateral 
central pain is most probably associated with lesions of the lateral posterior thalamus mainly affecting spinothalamocortical tracts neurons implicated in pain perception. The ipsilateral central central pain could be associated with the edema observed in the contralateral non-lesioned basal ganglia and thalamus. Bilateral central pain occurred only when massive lesions were induced. Finally findings support the alleviating effect of gabapentin on both allodynia and hyperalgesia in this model suggesting that it may a a treatment for central pain originating from thalamic lesions.

\section{ACKNOWLEDGEMENT}

We would like to thank Marie-Therèse Parent for the preparation of the figures and Guy Beauchamp for statistical analyses.

\section{REFERENCES}

Andersen, G., V. Vestergaard, M. Ingeman-Nielsen and T.S. Jensen,1995. Incidence of central post-stroke pain. Pain, 6: 187-193. DOI: 10.1016/03043959(94)00144-4

Anderson, T.E., 1982. A controlled pneumatic technique for experimental spinal cord contusion. J. Neurosci. Methods, 6: 327-333. DOI: 10.1016/0165-0270(82)90033-4

Beaudry, F., A. Ross, P.P. Lema and P. Vachon, 2010. Pharmacokinetics of vanillin and its effects on mechanical hypersensitivity in a rat model of neuropathic pain. J. Phytotherap, 24: 525-530. PMID: 19655294

Bennett, A.D., K.M. Chastain and C.E. Hulsebosch, 2000. Alleviation of mechanical and thermal allodynia by CGRP (8-37) in a rodent model of chronic central pain. Pain, 86: 163-175. DOI: 10.1016/S0304-3959(00)00242-6

Bowsher, D., 1996. Central pain, clinical and physiological characteristics. J. Neurol. Neurosurg. Psychiatr., 61: 62-69. DOI: 10.1136/jnnp.61.1.62

Bowsher, D., G. Leijon and K.A. Thuomas, 1998. Central poststroke pain, correlation of MRI with clinical pain characteristics and sensory abnormalities. Neurology, 51: 1352-1358.

Cassinari, V. and C.A. Pagni, 1969. Central Pain: A Neurosurgical Survey. 1st Edn., Harvard University Press, Cambridge, ISBN-10: 0674105400, pp: 102.

CCAC, 1993. Guide to the Care and Use of Experimental Animals. 2nd Edn., Canadian Council on Animal Care, Ottawa.
Christensen, M.D. and C.E. Hulsebosch, 1997.Chronic central pain after spinal cord injury. J. Neurotrauma, 14: 517-537. DOI: 10.1089/neu.1997.14.517

Christensen, M.D., Everhart, J.T. Pickelman and C.E. Hulsebosch, 1996. Mechanical and thermal allodynia in chronic central pain following spinal cord injury. Pain, 68: 97-107. DOI: 10.1016/S0304-3959(96)03224-1

Craig, D., 1998. A new version of the thalamic disinhibition hypothesis of central pain. Pain Forum, 7: 1-14. DOI: 10.1016/S10823174(98)70004-2

Dukes, J., 1992. Hypertension, a review of the mechanisms, manifestations and management. J. Small Anim. Pract., 33: 119-129. DOI: 10.1111/j.1748-5827.1992.tb01093.x

Dworkin, R.H., A.B. O’Connor, M. Backonja, J.T. Farrar and N.B. Finnerup et al., 2007. Pharmacologic management of neuropathic pain: Evidence-based recommendations. Pain, 132: 237251. DOI: 10.1016/j.pain.2007.08.033

Ferland, C., D. Veilleux-Lemieux, and P. Vachon, 2007. Effects of buprenorphine on intracerebral collagenase-induced hematoma in Sprague-Dawley rats. J. Am. Assoc. Lab. Anim. Sci., 46: 13-16.

Finnie, J.W. and P.C. Blumberg, 1990. Traumatic brain injury. Vet. Path., 39: 670-689.

Fishman, S., J.C. Ballantyne and J.P. Rathmell, 2009. Bonica's Management of Pain. 4th Edn., Lippincott Williams and Wilkins, Philadelphia, ISBN: 0781768276 , pp: 1661.

Garcin, R., 1968. Thalamic Syndrome and Pain Central Origin. In: Pain, Soulairac, A., J. Cahn and J. Charpentier (Eds.). Academic Press, London, pp: 521-541.

Gorman, A.L., C.G. Yu, G.R. Ruenes, L. Daniels and R.P. Yezierski, 2001. Conditions affecting the onset, severity and progression of spontaneous pain-like behavior after excitotoxic spinal cord injury. J. Pain, 2: 229-240.

Graham, D.I. and T.A. Gennarelli, 1997. Trauma. In: Neuropathology, Graham, D.I. and P.L. Lantos (Eds.). Greenfield's Neuropathology, Hodder Arnold, London, pp: 167-262.

Greenberg, J., P.E. McKeever and J.D. Balentine, 1978. Lysosomal activity in experimental spinal cord trauma, An ultrastructural cytochemical evaluation. Surg. Neurol., 9: 361-364.

Groothuis, D.R., G.W. Duncan and C.M. Fisher, 1977. The human thalamocortical sensory path in the internal capsule, evidence from a small capsular hemorrhage causing a pure sensory stroke. Ann. Neurol., 12: 328-331. DOI: 10.1002/ana.410020412 
Guenette, S.A., J.F. Marier, F. Beaudry and P. Vachon, 2007. Pharmacokinetics of eugenol and its effects on thermal hypersensitivity in rats. Eur. J. Pharmacol., 562: 60-67. DOI: 10.1016/j.ejphar.2007.01.044

Hansen, P., 2004. Post-stroke pain case study: Clinical characteristics, therapeutic options and long-term follow-up. Eur. J. Neurol., 11: 22-30. DOI: $10.1111 / \mathrm{j} .1471-0552.2004 .00793 . \mathrm{X}$

Hao, J.X., X.J. Xy, H. Aldskogius, A. Sieger and Z. Wiesenfeld-Hallin, 1992. Photochemically induced transient spinal ischemia induces behavioral hypersensitivity to mechanical and cold stimuli, but not to noxious-heat stimuli, in the rat. Exp. Neurol., 118: 187-194.

Kandel, E.R., J.H. Schwartz and T.M. Jessell, 1991. Principles of Neural Science. 3rd Edn., Elsevier, New York, ISBN: 0444009442, pp: 1135.

Kim, J.S., 1998. Delayed-onset ipsilateral sensory symptoms in patients with central post-stroke pain. Eur. Neurol., 40: 201-206. DOI: $10.1159 / 000007980$

Kim, J.S., 2003. Central post-stroke pain or paresthesia in lenticulo-capsular hemorrhages. Neurology, 61: 679-682.

Kumar, B., J. Kalita, G. Kumar and U.K. Misra, 2009. Central post-stroke pain: A review of patthophysiology and treatment. Pain Med., 108: 1645-1657. PMID: 19372350

Leijon, G., J. Boivie and I. Johansson, 1989. Central post-stroke pain-neurological symptoms and pain characteristics. Pain, 36: 13-26. PMID: 2919091

Lema, P.P., C. Girard and P. Vachon, 2004. Evaluation of dexamethasone for the treatment of intracerebral hemorrhage using a collagenase-induced intracerebral hematoma model in rats. J. Vet. Pharmacol. Therap., 27: 321-328. DOI: $10.1111 / \mathrm{j} .1365-2885.2004 .00597 . \mathrm{x}$

Lema, P.P., C. Girard and P. Vachon, 2005. High doses of methylprednisolone are required for the treatment of collagenase-induced intracerebral hemorrhage in rats. Can. J. Vet. Res., 6: 253-259. PMID: 16479722

Nakazato-Imasato, E. and Y. Kurebayashi, 2009. Pharmacological characteristics of the hindpaw weight bearing difference induced by chronic constriction injury of the sciatic nerve in rats. Life Sci, 84: 622-626. PMID: 9236885

Paxinos, G. and C. Watson, 1998. The Rat Brain in Stereotaxic Coordinates. 4th Edn., Academic Press, San Diego, ISBN-10: 0125476175, pp: 256.
Platt, S.R. and L. Garosi, 2003. Canine cerebrovascular disease, Do dogs have stroke? J. Am. Vet. Hosp. Assoc., 39: 337-342.

Reilly, P. and R. Bullock, 1997. Head Injury: Pathophysiology and Management of Severe Closed Injury. 1st Edn., Hodder Arnold Publishers, London, ISBN-10: 0412585405, pp: 500.

Rivlin, A.S. and C.H. Tator, 1978. Effect of duration of acute spinal cord compression in a new acute cord injury model in the rat. Surg. Neurol., 10: 38-43. PMID: 684604

Rosenberg, G.A., S. Mun-Bryce, M. Wesley and M. Kornfeld, 1990. Collagenase-induced cerebral hemorrhage in rats. Stroke, 21: 801-807.

Siddal, P.J., J.M. McClelland., S.B. Rutkowski and M.J. Cousins, 2003. A longitudinal study of the prevalence and characteristics of pain in the first 5 years following spinal cord injury. Pain, 103: 249257.

Tarlov, I.M., 1972. Acute spinal cord compression paralysis. J. Neurosurg., 36: 10-20. DOI: 10.3171/jns.1972.36.1.0010

Thomas, W.B., 1996. Cerebrovascular disease. Vet. Clinics North Am., Small. Anim. Pract., 26: 925943.

Vachon, P., R. Masse and R. Gibbs, 2004. Substance P and neurotensin are upregulated in the lumbar spinal cord of neuropathic animals. Can. J. Vet. Res., 69: 86-92.

Wall, P.D. and R. Melzack, 2001. Textbook of Pain. 4th Edn., Science Pr., Beijing, ISBN: 7030091469, pp. 1588.

Wasserman, J.K. and P.D. Koeberle, 2009. Development and characterization of a hemorrhagic rat model of central post-stroke pain. Neurosc., 161: 173-183. PMID: 19324079

Watson, B.D., R. Prado, W.D. Dietrich, M.D. Ginsberg and B.A. Green, 1986. Photochemically induced spinal cord injury in rat. Brain Res., 367: 296-300.

Widar, M., L. Samuelsson, S.K. Trevenious and G. Ahstrom, 2002. Long term pain condition after stroke. J. Rehab. Med. 34: 165-170.

Wilcox, G.L., 1988. Pharmacological studies of grooming and scratching behaviour elicited by spinal substance $\mathrm{P}$ and excitatory amino acids. Ann. New York Acad. Sci., 525: 228-236. PMID: 2455461 